\title{
Analyzing of singlet fermionic dark matter via the updated direct detection data
}

\author{
M. M. Ettefaghi ${ }^{\mathrm{a}}$, R. Moazzemi ${ }^{\mathrm{b}}$ \\ Department of Physics, University of Qom, Ghadir Blvd., Qom 371614-611, Islamic Republic of Iran
}

Received: 24 March 2017 / Accepted: 5 May 2017 / Published online: 23 May 2017

(C) The Author(s) 2017. This article is an open access publication

\begin{abstract}
We revisit the parameter space of singlet fermionic cold dark matter model in order to determine the role of the mixing angle between the standard model Higgs and a new singlet one. Furthermore, we restudy the direct detection constraints with the updated and new experimental data. As an important conclusion, this model is completely excluded by recent XENON100, PandaX II and LUX data.
\end{abstract}

\section{Introduction}

There exist several pieces of evidence that indicate the highest fraction of matter in the universe is composed of unknown particles called dark matter (DM) (see [1,2]). The baryonic matter contributes only less than $5 \%$ of the universe content. While the standard model (SM) is very successful in the experimental tests, it does not predict any appropriate candidate for DM. Hence, many authors have been convinced that we need a model beyond the SM. The evidence hints that the DM candidates should be mostly stable, non-baryonic, massive, non-relativistic and have insignificant or very weak interactions with other particles (see [3] for a discussion of the conditions of DM candidates and their properties). The DM particles with these properties are often called cold DM (CDM) or weakly interacting massive particles (WIMPs). Since no signal, predicted by any theory beyond SM, has been confirmed experimentally, it is reasonable to consider the most minimal extension of the SM to explain DM. Singlet fermionic CDM (SFCDM) is a minimal extension of the SM which proposes a singlet fermion as an appropriate candidate for CDM [4-7].

One can achieve a renormalizable theory for SFCDM if the $\mathrm{SM}$ is extended by a singlet fermion as CDM and a singlet scalar Higgs boson as a mediator. For the SFCDM masses below $100 \mathrm{GeV}$, the relic abundance constraint and the direct

\footnotetext{
a e-mail: mettefaghi@qom.ac.ir

be-mail: r.moazzemi@qom.ac.ir
}

detection bounds have been studied in $[5,6]$. An almost comprehensive study of the parameter space of SFCDM has been performed in [7]. The SFCDM annihilation into two photons under the relic abundance constraint has been obtained and compared with Fermi-Lat bounds for masses below $200 \mathrm{GeV}$ in [8]. From the Higgs coupling measurements, the mixing angle is constrained at $95 \%$ CL to be $\sin \theta \lesssim 0.4$ [9], independent of the second Higgs mass. The analysis of Ref. [10], by the electroweak precision tests, implies slightly stronger constraints in the relevant mass range; for example one finds $\sin \theta \leq 0.32$ for the second Higgs mass about $750 \mathrm{GeV}$ at 95\% CL. In addition, for this mass of the Higgs, it has been shown that $\sin \theta$ is constrained to be less than 0.1 , and this constraint is also put on any scenario where the new scalar is somehow involved in electroweak symmetry breaking [11]. In this paper, we restudy the parameter space of the SFCDM, focusing on the role of the Higgs mixing angle and compare our results with latest experimental data. We take the SM and singlet Higgs mass to be 125 and $750 \mathrm{GeV}$, respectively. The former is fixed by earlier ATLAS [19] and CMS [20] results. For the latter, due to the above statements for the Higgs mixing angle, we choose $750 \mathrm{TeV}$ as an interesting mass. ${ }^{1}$ Of course, as we shall state in Sect. 3.1, for the other masses between the range about $500-1000 \mathrm{GeV}$ our general results and discussions do not get altered.

Furthermore, there are several experiments which report the measured cross section for direct detection of dark matter, recently, such as the XENON100, LUX, COUPP, PICO, EDELWIESS II, PandaX II and Darkside Collaborations. In this paper, using the most updated direct detection data reported by some of these experiments and considering the issues on the mixing angle mentioned above, we reanalyze

\footnotetext{
${ }^{1}$ It is also notable that an excess in the diphotons events with the invariant mass of about $750 \mathrm{GeV}$ has been reported by ATLAS [12] and CMS [13] based on data collected in 2015, though the analyzes based on data collected in $2016[14,15]$ show no significant excess over the SM predictions.
} 
the parameter space by imposing the relic abundance condition. We shall see that the entire parameter space is excluded by XENON100 [21], PandaX II [22], and LUX [23].

We have organized the paper as follows: in Sect. 2 the renormalizable model for a SFCDM is briefly reviewed. In Sect. 3, we obtain the coupling constant by imposing the relic abundance condition, then we calculate the scattering cross section of SFCDM from the nucleon and explore the parameter space using the most recent direct detection data. Finally, we summarize our discussion and conclusions in the last section.

\section{The model}

The most minimal extension of the SM, including a CDM candidate, is achieved by adding a gauge singlet fermion. We can consider the singlet fermion to play the dark matter role (SFCDM) provided that it has a very weak interaction with the SM particles because it must respect the relic abundance condition. To accommodate this in a renormalizable manner, a singlet Higgs $S$, in addition to the usual Higgs doublet, is needed as mediator between SFCDM and the SM particles [5,7]. The Lagrangian for the SFCDM model can be decomposed as follows:

$\mathcal{L}_{\mathrm{SFCDM}}=\mathcal{L}_{\mathrm{SM}}+\mathcal{L}_{\text {hid }}+\mathcal{L}_{\text {int }}$,

where $\mathcal{L}_{\mathrm{SM}}$ is the SM Lagrangian and $\mathcal{L}_{\text {hid }}$ denotes the hidden sector Lagrangian,

$\mathcal{L}_{\text {hid }}=\mathcal{L}_{\psi}+\mathcal{L}_{\mathrm{S}}-g_{s} \bar{\psi} \psi S$.

Here, $\mathcal{L}_{\psi}$ and $\mathcal{L}_{\mathrm{S}}$ are the free Lagrangians of SFCDM,

$\mathcal{L}_{\psi}=\bar{\psi}\left(i \not \partial-m_{\psi_{0}}\right) \psi$,

and the singlet Higgs,

$\mathcal{L}_{\mathrm{S}}=\frac{1}{2}\left(\partial_{\mu} S\right)\left(\partial^{\mu} S\right)-\frac{m_{0}^{2}}{2} S^{2}-\frac{\lambda_{3}}{3 !} S^{3}-\frac{\lambda_{4}}{4 !} S^{4}$.

The last term in Eq. (2) is due to the interaction between the SFCDM and singlet Higgs with coupling constant $g_{s}$. In Eq. (1), $\mathcal{L}_{\text {int }}$ is related to the interaction between the new singlet Higgs and the SM doublet one,

$\mathcal{L}_{\text {int }}=-\lambda_{1} H^{\dagger} H S-\lambda_{2} H^{\dagger} H S^{2}$.

We have $\langle H\rangle=\frac{1}{\sqrt{2}}\left(\begin{array}{c}0 \\ v_{0}\end{array}\right)$ and $\langle S\rangle=x_{0}$, with $v_{0}$ and $x_{0}$ being the vacuum expectation values (VEV) of the SM Higgs and singlet Higgs, respectively. We define the fields $h$ and $s$ as the fluctuation around the VEVs of them. Therefore, after symmetry breaking we have

$H=\frac{1}{\sqrt{2}}\left(\begin{array}{c}0 \\ h+v_{0}\end{array}\right)$, and

$S=s+x_{0}$.

We can obtain the mass eigenstates by diagonalizing the mass matrix as follows:

$h_{1}=\sin \theta s+\cos \theta h$,

$h_{2}=\cos \theta s-\sin \theta h$,

where $\theta$ is a mixing angle which depends on the parameters of the Lagrangian (1). One naturally expects that $|\cos \theta|>$ $\frac{1}{2}$, so that $h_{1}$ is the SM Higgs-like scalar, while $h_{2}$ is the singlet-like one. The singlet fermion has mass $m_{\psi}=m_{\psi_{0}}+$ $g_{S} x_{0}$, which is an independent parameter in the model. The VEV of our singlet Higgs, $x_{0}$, is completely determined by minimization of the total potential (including SM and singlet Higgs potentials) as follows:

$x_{0}=-\frac{1}{4 v \lambda_{2}}\left[\left(m_{h_{1}}^{2}+m_{h_{2}}^{2}-4 v^{2} \lambda_{0}\right) \tan 2 \theta+2 v \lambda_{1}\right]$,

where $\lambda_{0}$ is the self-interaction coupling constant of the SM Higgs. There are seven independent parameters, in addition to the SM ones, in this model: $\left\{m_{\psi}, g_{S}, m_{0}, \lambda_{1}, \lambda_{2}, \lambda_{3}, \lambda_{4}\right\}$. After spontaneous symmetry breaking we encounter a new set of parameters, $m_{\psi}, g_{S}$, second Higgs mass $m_{h_{2}}, \lambda_{1}, \lambda_{2}$, $\lambda_{3}, \lambda_{4}$ and the mixing angle between Higgs bosons $\theta$, which is not an independent parameter.

\subsection{The cross section}

In the SFCDM model, at tree level, pairs of singlet fermions can annihilate into SM particles, including pairs of massive fermions and gauge bosons, and also two and three Higgs bosons. We have listed the corresponding Feynman diagrams in Fig. 1. These diagrams are at leading order, so we should respect the perturbation criteria in our calculations.

We have calculated the corresponding cross section of this annihilation process. According to Fig. 1, while the annihilations into the fermions and gauge bosons proceed only through the $s$-channel, the annihilation into Higgs bosons occurs via the $s$-, $t$ - and $u$-channels. The total annihilation cross section times the relative velocity $v$ can be written as follows:

$\sigma v_{\mathrm{ann}}=\sigma v_{\mathrm{SM}}+\sigma v_{2 \mathrm{Higgs}}+\sigma v_{3 \mathrm{Higgs}}$,

where the $\sigma v_{\mathrm{SM}}$ is given by

$$
\begin{aligned}
\sigma v_{\mathrm{SM}}= & \frac{\left(g_{s} s_{1} s_{2}\right)^{2}}{16 \pi}\left(1-\frac{4 m_{\psi}^{2}}{s}\right) \\
& \times\left(\sum_{j=1,2} \frac{1}{d_{j}}-\frac{2\left(s-m_{h_{1}}^{2}\right)\left(s-m_{h_{2}}^{2}\right)+2 m_{h_{1}} m_{h_{2}} \Gamma_{h_{1}} \Gamma_{h_{2}}}{d_{1} d_{2}}\right)
\end{aligned}
$$



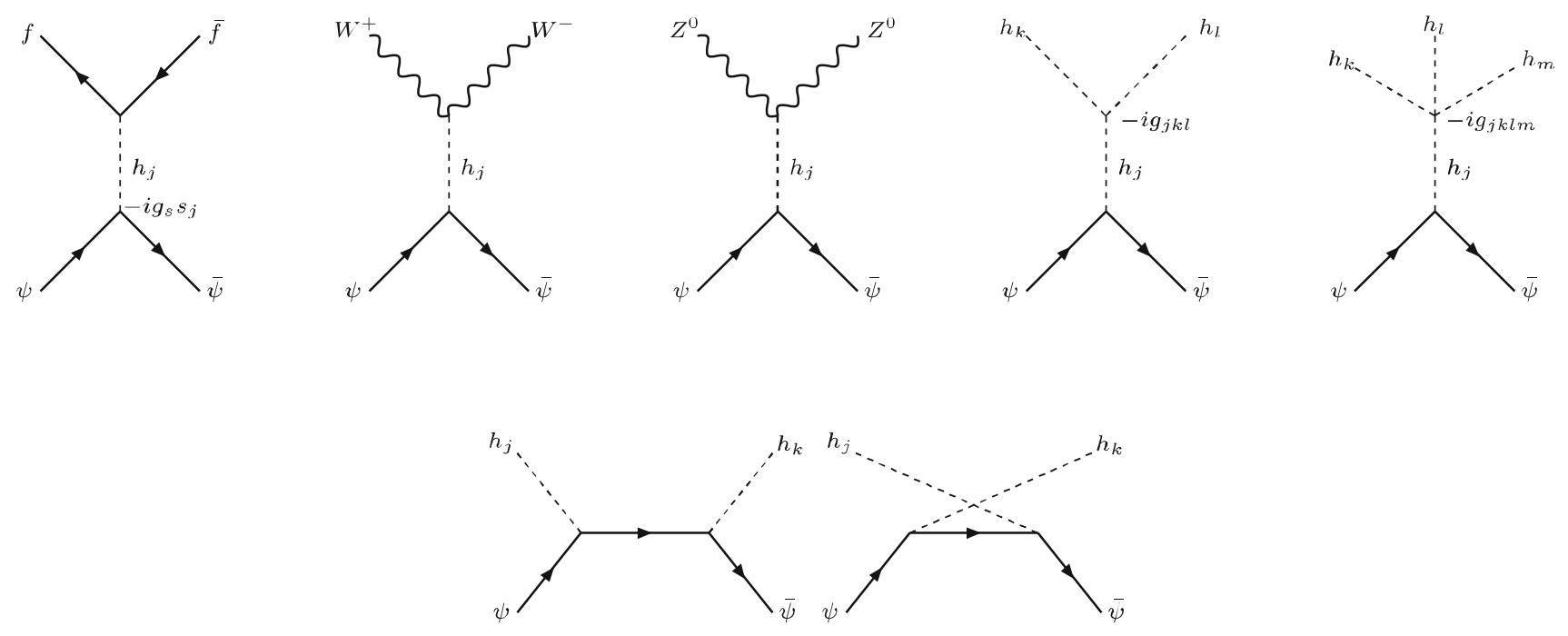

Fig. 1 The Feynman diagrams for the annihilation of singlet fermion pairs into SM particles, two and three Higgs bosons at tree level. The vertex factor of three (four) Higgs boson lines, $-i g_{i j k}\left(-i g_{i j k l}\right)$, is symmetric under permutations of their subscripts. For three Higgs bosons in final state only the dominant Feynman diagrams are shown. Obviously, the first row is due to the $s$-channel, while the second row indicates the $t$ - and $u$-channels

$$
\begin{aligned}
& \times\left[\sum_{f \text { (fermions) }} 2 \lambda_{f} s\left(\frac{m_{f}}{v_{0}}\right)^{2}\left(1-\frac{4 m_{f}^{2}}{s}\right)^{3 / 2}\right. \\
& \left.+\sum_{w=W^{+}, W^{-}, Z^{0}} 2\left(\frac{m_{w}^{2}}{v_{0}}\right)^{2}\left(2+\frac{\left(s-2 m_{w}^{2}\right)^{2}}{4 m_{w}^{4}}\right) \sqrt{1-\frac{4 m_{w}^{2}}{s}}\right],
\end{aligned}
$$

where $\lambda_{f}$ is 3 (1) for quarks (leptons), $\Gamma_{h_{j}}$ refers to the decay widths of $h_{j}$ and $d_{j}=\left(s-m_{h_{j}}^{2}\right)^{2}+m_{h_{j}}^{2} \Gamma_{h_{j}}^{2}(j=1,2)$. Here, we have used the abbreviations $s_{1} \equiv \sin \theta$ and $s_{2} \equiv$ $\cos \theta$. The last two terms in Eq. (9) are the annihilation cross sections into two and three Higgs bosons, respectively. To obtain these cross sections we should derive $g_{j k l}$ and $g_{j k l m}$ corresponding to the vertex factors of them. For $j \neq k$ we get

$$
\begin{aligned}
g_{j j j}= & \frac{1}{3}\left\{6(-1)^{j} v_{0} s_{k}\left(\lambda_{2} s_{j}^{2}+\lambda_{0} s_{k}^{2}\right)\right. \\
& \left.-s_{j}\left[s_{j}^{2}\left(\lambda_{3}+\lambda_{4} x_{0}\right)+3 \lambda_{1} s_{k}^{2}\right]\right\}, \\
& +s_{j}^{2} s_{k}\left[9 \lambda_{1}-4 \lambda_{3}+2\left(9 \lambda_{2}-2 \lambda_{4}\right) x_{0}\right] \\
& \left.-\left(3 s_{k}^{3}+s_{k}\right)\left(\lambda_{1}+2 \lambda_{2} x_{0}\right)\right\}, \\
g_{j j j j}= & -12 \lambda_{2} s_{1}^{2} s_{2}^{2}-\lambda_{4} s_{j}^{4}-6 \lambda_{0} s_{k}^{4}, \\
g_{1122}= & \frac{1}{8}\left\{[\cos (4 \theta)-1]\left(\lambda_{4}+6 \lambda_{0}\right)-4 \lambda_{2}[3 \cos (4 \theta)+1]\right\}, \\
g_{j j j k}= & s_{2} s_{1}\left(6 \lambda_{2}\left(s_{j}^{2}-s_{k}^{2}\right)-\lambda_{4} s_{j}^{2}+6 \lambda_{0} s_{k}^{2}\right) .
\end{aligned}
$$

Note that $g_{j k l}$ and $g_{j k l m}$ are symmetric under permutation of their subscripts and $j, k, l, m=1,2$. Therefore, one can derive the annihilation cross section into two Higgs bosons as follows:

$$
\begin{aligned}
& \sigma v_{2 H i g g s} \\
& =\frac{g_{s}^{2}}{16 \pi}\left(1-\frac{4 m_{\psi}^{2}}{s}\right)\left\{-\frac{4 g_{s}^{2} s_{1}^{2} s_{2}^{2}}{y\left(y^{2}-1\right)\left(-m_{h_{1}}^{2}-m_{h_{2}}^{2}+s\right)^{2}}\right. \\
& \times\left\{\left(-m_{h_{1}}^{2}-m_{h_{2}}^{2}+s\right)^{2} y^{3}+\left[-32 m_{\psi}^{4}+8\left(m_{h_{1}}^{2}+m_{h_{2}}^{2}\right) m_{\psi}^{2}\right.\right. \\
& \left.-m_{h_{1}}^{4}-\left(m_{h_{2}}^{2}-s\right)^{2}-m_{h_{1}}^{2}\left(4 m_{h_{2}}^{2}-2 s\right)\right] y \\
& +\left(y^{2}-1\right) \tanh ^{-1} y\left[32 m_{\psi}^{4}+8\left(m_{h_{1}}^{2}+m_{h_{2}}^{2}-2 s\right) m_{\psi}^{2}\right. \\
& \left.\left.-m_{h_{1}}^{4}-\left(m_{h_{2}}^{2}-s\right)^{2}+2 m_{h_{1}}^{2}\left(s-2 m_{h_{2}}^{2}\right)\right]\right\} \\
& -\frac{8 g_{s} m_{\psi} s_{1} s_{2}}{d_{1} d_{2}}\left[\frac{\tanh ^{-1} y\left(8 m_{\psi}^{2}-m_{h_{1}}^{2}-m_{h_{2}}^{2}-s\right)}{y\left(-m_{h_{1}}^{2}-m_{h_{2}}^{2}+s\right)}-1\right] \\
& \times\left[d_{2} g_{112}\left(s-m_{h_{1}}^{2}\right) s_{1}+d_{1} g_{212}\left(s-m_{h_{2}}^{2}\right) s_{2}\right] \\
& +\sqrt{\frac{\left(-m_{h_{1}}^{2}-m_{h_{2}}^{2}+s\right)^{2}-4 m_{h_{1}}^{2} m_{h_{2}}^{2}}{s^{2}}}\left[\frac{2 g_{112} g_{212} s_{1} s_{2}}{d_{1} d_{2}}\right. \\
& \left.\times\left[\left(s-m_{h_{1}}^{2}\right)\left(s-m_{h_{2}}^{2}\right)+m_{h_{1}} m_{h_{2}} \Gamma_{h_{1}} \Gamma_{h_{2}}\right]+\sum_{j=1,2} \frac{g_{j 12}^{2} s_{j}}{d_{j}}\right] \\
& +\frac{1}{2} \sum_{k=1,2}\left[\frac { g _ { s } ^ { 2 } s _ { k } ^ { 4 } } { x _ { k } ( x _ { k } ^ { 2 } - 1 ) ( s - 2 m _ { h _ { k } } ^ { 2 } ) ^ { 2 } } \left[4 x _ { k } \left(32 m_{\psi}^{4}-16 m_{h_{k}}^{2} m_{\psi}^{2}\right.\right.\right. \\
& \left.+6 m_{h_{k}}^{4}+s^{2}-4 s m_{h_{k}}^{2}-\left(s-2 m_{h_{k}}^{2}\right)^{2} x_{k}^{2}\right) \\
& -4\left(x_{k}^{2}-1\right) \tanh ^{-1} x_{k}\left(32 m_{\psi}^{4}+16\left(m_{h_{k}}^{2}-s\right)\right. \\
& \left.\left.\times m_{\psi}^{2}-6 m_{h_{k}}^{4}-s^{2}+4 s m_{h_{k}}^{2}\right)\right] \\
& +\sqrt{1-\frac{4 m_{h_{k}}^{2}}{s}}\left(\frac { 2 g _ { 1 k k } g _ { 2 k k } s _ { 1 } s _ { 2 } } { d _ { 1 } d _ { 2 } } \left[\left(s-m_{h_{1}}^{2}\right)\left(s-m_{h_{2}}^{2}\right)\right.\right.
\end{aligned}
$$




$$
\begin{aligned}
& \left.\left.+m_{h_{1}} m_{h_{2}} \Gamma_{h_{1}} \Gamma_{h_{2}}\right]+\sum_{j=1,2} \frac{g_{j k k}^{2} s_{j}}{d_{j}}\right) \\
& -\frac{8\left(g_{s} m_{\psi} s_{k}^{2}\right)}{d_{1} d_{2}}\left(\frac{\tanh ^{-1} x_{k}\left(-8 m_{\psi}^{2}+2 m_{h_{k}}^{2}+s\right)}{\left(2 m_{h_{k}}^{2}-s\right) x_{k}}-1\right) \\
& \left.\left.\times \sum_{j=1,2} g_{j k k}\left(s-m_{h_{j}}^{2}\right) s_{j} d_{j}\right]\right\},
\end{aligned}
$$

where

$$
x_{k}=\sqrt{1-\frac{4 m_{\psi}^{2}}{s}} \sqrt{1-\frac{4 m_{h_{k}}^{2}}{s}} /\left(1-\frac{2 m_{h_{k}}^{2}}{s}\right)
$$

and
$x_{F}=\ln \left(\frac{m_{\psi}}{2 \pi^{3}} \sqrt{\frac{45 M_{\mathrm{Pl}}}{2 g_{*} x_{F}}}\left\langle\sigma v_{\mathrm{ann}}\right\rangle\right)$

where $g_{*}$ is for the effective degrees of freedom for the relativistic quantities in equilibrium [17] and $M_{\mathrm{Pl}}=1.22 \times$ $10^{19} \mathrm{GeV}$ is the Planck mass.

To study the allowed parameter space consistent with the relic abundance constraint obtained by WMAP observations [18], the SM Higgs boson mass is fixed to $125 \mathrm{GeV}$ according to the 2012 CMS and ATLAS results [19,20] and the other Higgs mass to $750 \mathrm{GeV}^{2}$ Although the variations of the $\lambda$ have no significant impact [7], we let them vary as far as perturbation theory is correct. To find the couplings $g_{s}$ which satisfy the relic density condition, we first inves-

$y=-\sqrt{1-\frac{4 m_{\psi}^{2}}{s}} \sqrt{\frac{m_{h_{1}}^{4}}{s^{2}}+\left(\frac{m_{h_{2}}^{2}}{s}+1\right)\left(1-\frac{2 m_{h_{1}}^{2}+m_{h_{2}}^{2}}{s}\right)} /\left(1-\frac{m_{h_{1}}^{2}+m_{h_{2}}^{2}}{s}\right)$.

Although the annihilation cross section into three Higgs bosons is suppressed due to its narrow phase space integral, to have a complete and more precise calculation we take it into account. For this term we have

$$
\begin{gathered}
\sigma v_{3 \mathrm{Higgs}}=\frac{2 g_{s}^{2}\left(s-4 m_{\psi}^{2}\right)}{1536 \pi^{3}} \sum_{k, l, m}\left(\sum_{j=1,2} \frac{g_{j k l m}^{2} s_{j}^{2}}{d_{j}}\right. \\
\left.+\frac{2 s_{1} s_{2} g_{1 k l m} g_{2 k l m}\left[\Gamma_{h_{1}} \Gamma_{h_{2}} m_{h_{1}} m_{h_{2}}+\left(s-m_{h_{1}}^{2}\right)\left(s-m_{h_{2}}^{2}\right)\right]}{d_{1} d_{2}}\right) .
\end{gathered}
$$

\section{Computations}

\subsection{The relic density}

The relic density $\Omega_{\psi} h^{2}$, defined as the ratio of the present density of particles to the critical density, is written as follows:

$\Omega_{\psi} h^{2} \approx \frac{\left(1.07 \times 10^{9}\right) x_{F}}{\sqrt{g_{*}} M_{\mathrm{Pl}}(\mathrm{GeV})\left\langle\sigma v_{\mathrm{ann}}\right\rangle}$,

where $\left\langle\sigma v_{\text {ann }}\right\rangle$ is the thermally averaged annihilation cross section times the relative velocity [16]:

$$
\begin{aligned}
\left\langle\sigma v_{\mathrm{ann}}\right\rangle= & \frac{1}{8 m_{\psi}^{4} T_{F} K_{2}^{2}\left(\frac{m_{\psi}}{T_{F}}\right)} \\
& \times \int_{4 m_{\psi}^{2}}^{\infty} d s \sigma_{\mathrm{ann}}(s)\left(s-4 m_{\psi}^{2}\right) \sqrt{s} K_{1}\left(\frac{\sqrt{s}}{T_{F}}\right),
\end{aligned}
$$

with $K_{1,2}(x)$ being the modified Bessel functions. Here $x_{F}=$ $m_{\psi} / T_{F}$ is the inverse freeze-out temperature, which can be determined by the following iterative equation: tigate about 25,000 sample models randomly in the whole parameter space. Namely, in addition to the $\lambda$, we take $\theta$ and $m_{\psi}$ to be free. In the other two investigations, each of which concerned with 10,000 sample models, we set $\theta=0.1$ and $\theta=0.01$. We collect all of these three data sets in Fig. 2 . Using our first data set, we also illustrate the role of the mixing angle $\theta$ by the contour plot of Fig. 3. This figure shows that for $\theta<0.1$ there is only a mass region between about $700-1000 \mathrm{GeV}$ as well as a narrow one about $350 \mathrm{GeV}$, where we get $g_{s}<1$ and therefore our perturbative analysis works self-consistently. For the other regions, although obtaining $g_{s}$ from the relic density is not consistent with perturbation theory, we necessarily conclude that $g_{s}>1$.

\subsection{Direct detection}

In this subsection, we investigate the consistency of SFCDM with the direct detection bounds. We use the following effective Lagrangian at the hadronic level to describe the scattering of SFCDM from a nucleon:

$\mathcal{L}_{\mathrm{eff}}=f_{\mathrm{p}}(\bar{\psi} \psi)(\overline{\mathrm{p}} \mathrm{p})+f_{\mathrm{n}}(\bar{\psi} \psi)(\overline{\mathrm{n}} \mathrm{n})$,

where $f_{\mathrm{p}}$ and $f_{\mathrm{n}}$ are the effective couplings of DM to protons and neutrons, respectively, and they are given by

$$
\frac{f_{\mathrm{p}, \mathrm{n}}}{m_{\mathrm{p}, \mathrm{n}}}=\sum_{q=u, d, s} f_{T q}^{(\mathrm{p}, \mathrm{n})} \frac{\alpha_{q}}{m_{q}}+\frac{2}{27} f_{T g}^{(\mathrm{p}, \mathrm{n})} \sum_{q=c, b, t} \frac{\alpha_{q}}{m_{q}}
$$

\footnotetext{
${ }^{2}$ From Ref. [7] one can see, for a wide range of masses (about 500$1000 \mathrm{TeV}$ ) the minimum of $g_{s}$ does not change seriously, so that the direct detection cross section cannot fall below the XENON100 bound (see Fig. 4).
} 


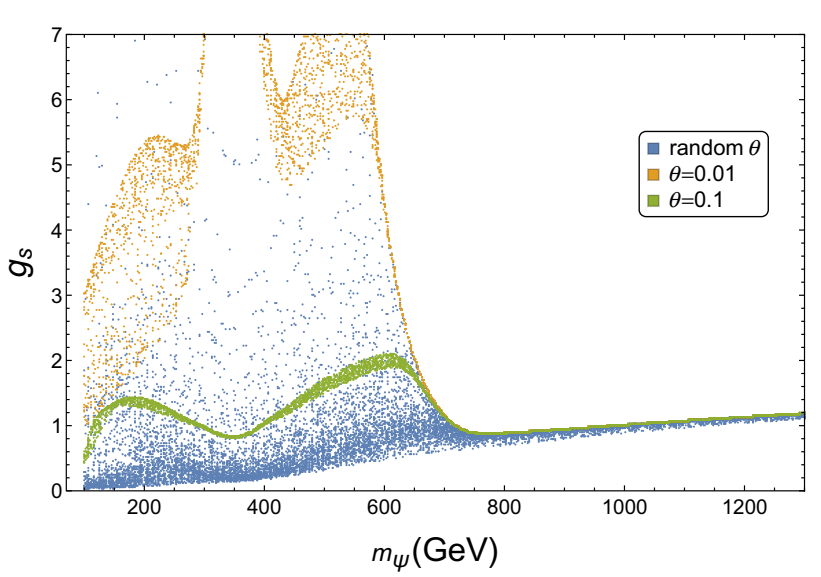

Fig. 2 The coupling $g_{s}$ in terms of the dark matter mass $m_{\psi}$ for three different types of choice of the mixing angle $\theta$

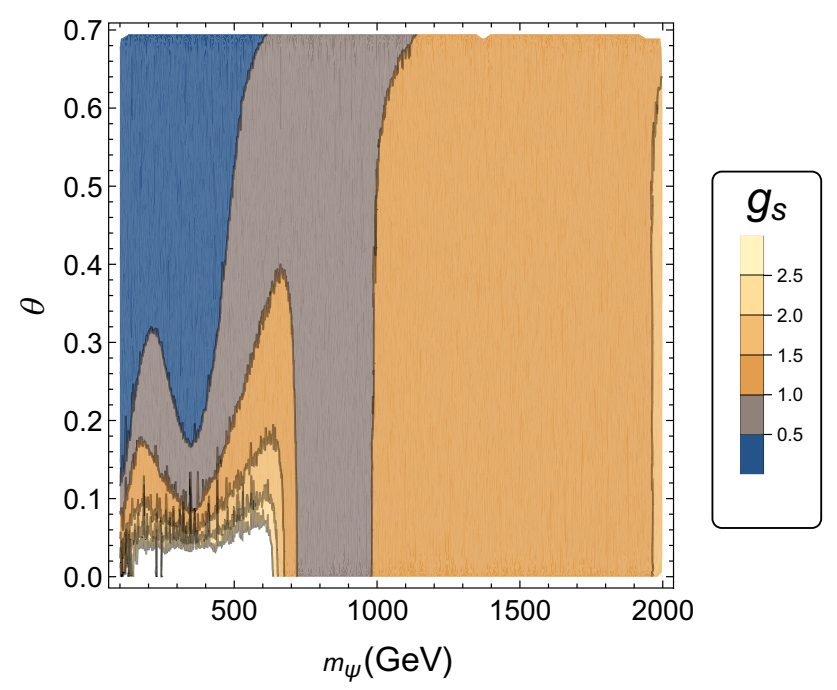

Fig. 3 The contour plot of the $\theta$ in terms of dark matter mass $m_{\psi}$; the color illustrates the change in coupling $g_{s}$

with the matrix elements $m_{\mathrm{p}, \mathrm{n}} f_{T q}^{(\mathrm{p}, \mathrm{n})} \equiv\left\langle\mathrm{p}, \mathrm{n}\left|m_{q} \bar{q} q\right| \mathrm{p}, \mathrm{n}\right\rangle$ for $q=u, d, s$ and $f_{T g}^{(\mathrm{p}, \mathrm{n})}=1-\sum_{q=u, d, s} f_{T q}^{(\mathrm{p}, \mathrm{n})}$. The numerical values of the hadronic matrix elements are given in [24]. Here, $\alpha_{q}$ is an effective coupling constant between SFCDM and quark $q$, in the following effective Lagrangian:

$\mathcal{L}_{\text {eff }}=\sum_{q} \alpha_{q} \bar{\psi} \psi \bar{q} q$

Since the scattering SFCDM and quarks proceeds through $t$ channel by intermediating a Higgs boson, $\alpha_{q}$ can be derived:

$\alpha_{q}=\frac{g_{s} \sin \theta \cos \theta m_{q}}{v_{0}}\left(\frac{1}{m_{h_{1}}^{2}}-\frac{1}{m_{h_{2}}^{2}}\right)$.
Consequently, the elastic spin-independent scattering cross section off a single nucleon becomes

$\sigma(\psi \mathrm{p} \rightarrow \psi \mathrm{p})=\frac{4 m_{\mathrm{r}}^{2}}{\pi} f_{\mathrm{p}}^{2}$,

where $m_{\mathrm{r}}=\left(\frac{1}{m_{\psi}}+\frac{1}{m_{\mathrm{p}}}\right)^{-1}$.

Using $g_{s}$ as obtained in the previous subsection for $\theta=$ 0.1 and 0.01 we plot the direct detection cross section in Fig. 4. We also compare our result with the new updated experimental data in this figure. The data which we have used here are from the XENON100 [21], PandaX II [22], LUX [23], PICO-60 [25] and Darkside-50 [26] Collaborations.

\section{Discussion and conclusions}

The most minimal and renormalizable extension of the SM, which introduces a singlet fermion as CDM candidate, is the SFCDM model. Namely, one adds a singlet fermion as CDM and a scalar as mediator to the SM content. A comprehensive analysis of this model has been given in [7]. However, the mixing angle between the SM Higgs and singlet scalar is constrained to be less than 0.1 [11]. Therefore, we have restudied the relevant parameter space to determine the role of the mixing angle. The SM Higgs boson mass is fixed to $125 \mathrm{GeV}$ according to the 2012 ATLAS [19] and CMS [20] reports and the other Higgs mass to $750 \mathrm{GeV}$ as we have explained in the main body of paper. In order to find the coupling $g_{s}$ which satisfies the relic density condition, we first investigate about 25,000 sample models randomly in the whole parameter space. In fact, in addition to the $\lambda$, we take $\theta$ and $m_{\psi}$ to be free. The data of this study is denoted by blue points in Fig. 2 . We see that $g_{s}$ tends to a unique value for $m_{\psi}$ larger than about $750 \mathrm{GeV}$. Two other investigations with fixed $\theta=0.1$ and $\theta=0.01$, each of which with 10,000 sample models, have been denoted in Fig. 2 by orange and green points, respectively. For more clarification, we illustrate the behavior of $g_{s}$ in terms of $m_{\psi}$ and $\theta$ through Fig. 3. We see that there exist limited regions $\left(300 \mathrm{GeV}<m_{\psi}<400 \mathrm{GeV}\right.$ and $700 \mathrm{GeV}<m_{\psi}<1000 \mathrm{GeV}$ ) in which $\theta<0.1$ and $g_{s}<1$. Furthermore, after deriving the spin-independent cross section of the elastic scattering of SFCDM from nucleon, we use the $g_{s}$ obtained from relic abundance condition to calculate and plot this cross section. It is illustrated through Fig. 4 in terms of $m_{\psi}$ for two various choices of $\theta$. We have compared our results with different experimental data. According to this figure, the entire parameter space is excluded by XENON100 [21], LUX [23] and PandaX II [22]. For more comparison, we have also shown the recent experiments PICO-60 [25] and DarkSide-50 [26] in this figure. 


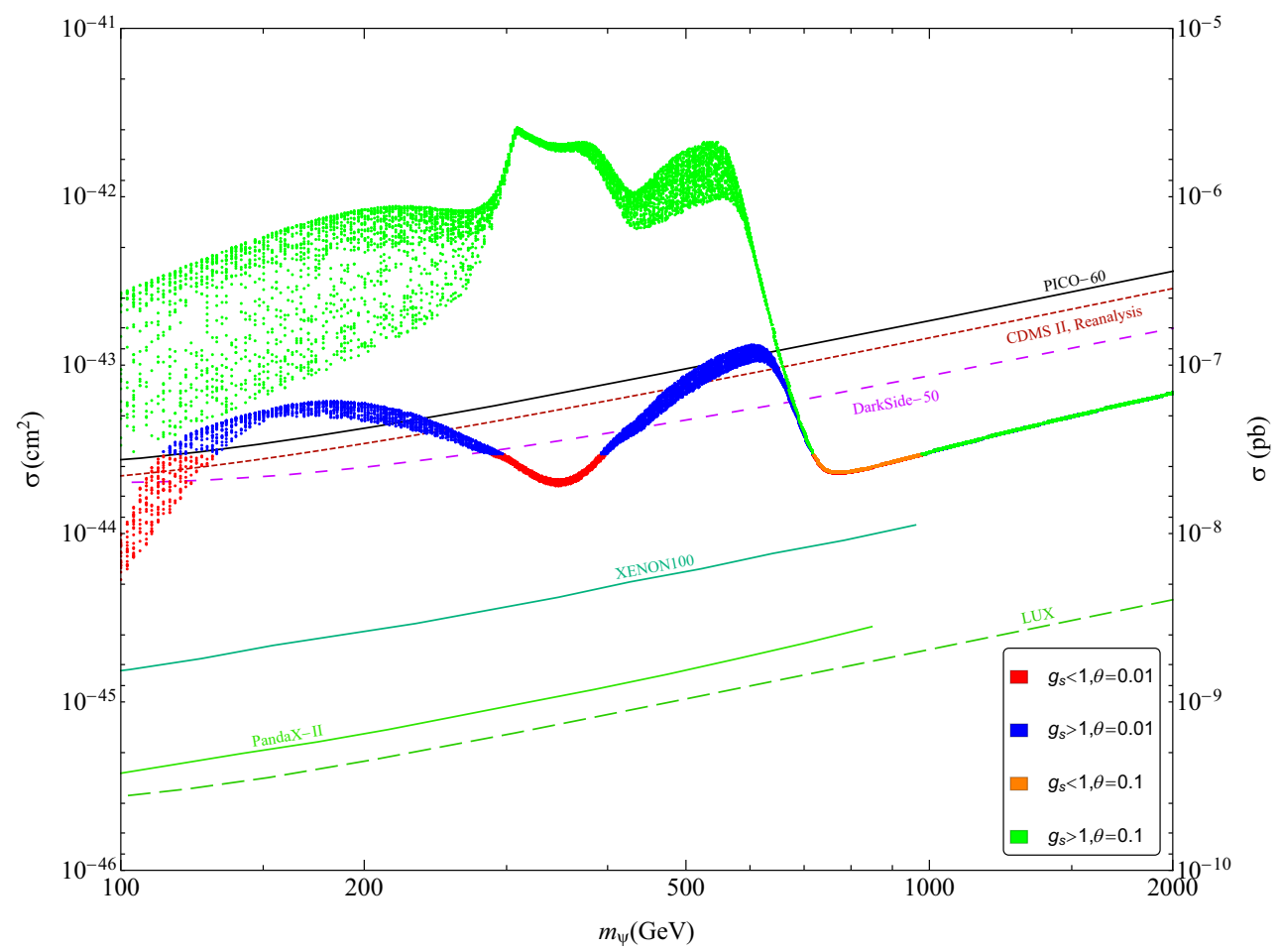

Fig. 4 The elastic scattering cross section off a nucleon in terms of $m_{\psi}$ for two different values of Higgs mixing angle; $\theta=0.01$ and $\theta=0.1$

Open Access This article is distributed under the terms of the Creative Commons Attribution 4.0 International License (http://creativecomm ons.org/licenses/by/4.0/), which permits unrestricted use, distribution, and reproduction in any medium, provided you give appropriate credit to the original author(s) and the source, provide a link to the Creative Commons license, and indicate if changes were made. Funded by SCOAP ${ }^{3}$.

\section{References}

1. G. Jungman, M. Kamionkowski, K. Griets, Supersymmetric dark matter. Phys. Rep. 267, 195 (1996)

2. G. Bertone, D. Hooper, J. Silk, Particle dark matter: evidence, candidates and constraints. Phys. Rep. 405, 279 (2005)

3. M. Taoso, G. Bertone, A. Masiero, Dark matter candidates: a tenpoint test. JCAP 0803, 022 (2008)

4. Y.G. Kim, K.Y. Lee, Minimal model of fermionic dark matter. Phys. Rev. D 75, 115012 (2007)

5. Y.G. Kim, K.Y. Lee, S. Shin, Singlet fermionic dark matter. JHEP 0805, 100 (2008)

6. S. Baek, P. Ko, W.-I. Park, E. Senaha, Search for the Higgs portal to a singlet fermionic dark matter at the LHC. JHEP 1211, 116 (2012)

7. Z. Bagherian, M.M. Ettefaghi, Z. Haghgouyan, R. Moazzemi, A new parameter space study of the fermionic cold dark matter model. JCAP 10, 033 (2014)

8. M.M. Ettefaghi, R. Moazzemi, Annihilation of singlet fermionic dark matter into two photons. JCAP 02, 048 (2013). arXiv: 1301.4892

9. ATLAS and CMS Collaboration, Measurements of the Higgs boson production and decay rates and constraints on its couplings from a combined ATLAS and CMS analysis of the LHC pp collision data at $\sqrt{s}=7$ and 8 TeV. JHEP 08, 045 (2016)
10. A. Falkowski, C. Gross, O. Lebedev, A second Higgs from the Higgs portal. JHEP 05, 057 (2015). arXiv:1502.01361

11. A. Falkowski, O. Sloneb, Tomer Volansky, Phenomenology of a $750 \mathrm{GeV}$ singlet. JHEP 02, 152 (2016). arXiv:1512.05777v3

12. ATLAS Collaboration, M. Aaboud, et al. Search for resonances in diphoton events at $\sqrt{s}=13 \mathrm{TeV}$ with the ATLAS detector. JHEP 09, 001 (2016). arXiv:1606.03833

13. CMS Collaboration, V. Khachatryan, et al. Search for resonant production of high-mass photon pairs in proton-proton collisions at $\sqrt{s}=8$ and 13 TeV. Phys. Rev. Lett. 117, 051802 (2016). arXiv: 1606.04093

14. ATLAS collaboration, Search for scalar diphoton resonances with $15.4 \mathrm{fb}^{-1}$ of data collected at $\sqrt{s}=13 \mathrm{TeV}$ in 2015 and 2016 with the ATLAS detector. Tech. Report ATLAS-CONF-2016 (2016)

15. CMS collaboration, Search for resonant production of high mass photon pairs using $12.9 \mathrm{fb}^{1}$ of proton-proton collisions at $\sqrt{s}=13$ $\mathrm{TeV}$ and combined interpretation of searches at 8 and $13 \mathrm{TeV}$. CMS PAS EXO-16-027 (2016)

16. P. Gondolo, G. Gelmini, Cosmic abundances of stable particles: improved analysis. Nucl. Phys. B 360, 145 (1991)

17. E.W. Kolb, M.S. Turner, The Early Universe (Addison Wesley, New York, 1990)

18. WMAP Collaboration, D.N. Spergel et al., Wilkinson microwave anisotropy probe (wmap) three year results: implications for cosmology. Astrophys. J. Suppl. 170, 377 (2007). arXiv:astro-ph/0603449

19. ATLAS Collaboration, G. Aad et al., Observation of a new particle in the search for the standard model Higgs boson with the ATLAS detector at the LHC. Phys. Lett. B 716, 1 (2012). arXiv:1207.7214

20. CMS Collaboration, S. Chatrchyan et al., Observation of a new boson at a mass of $125 \mathrm{GeV}$ with the CMS experiment at the LHC. Phys. Lett. B 716, 30 (2012). arXiv:1207.7235 
21. XENON100 Collaboration, E. Aprile, et al., XENON100 dark matter results from a combination of 477 live days. Phys. Rev. D 94, 122001 (2016)

22. PandaX-II Collaboration, A. Tan, et al., Dark matter results from first 98.7-days of data from the PandaX-II experiment. Phys. Rev. Lett. 117, 121303 (2016)

23. LUX Collaboration, D.S. Akerib et al., Results from a search for dark matter in LUX with 332 live days of exposure. Phys. Rev. Lett. 118, 021303 (2017)
24. J. Ellis, A. Ferstl, K.A. Olive, Re-evaluation of the elastic scattering of supersymmetric dark matter. Phys. Lett. B 481, 304 (2000)

25. PICO Collaboration, C. Amole et al., Dark matter search results from the PICO-60 CF 3 I bubble chamber. Phys. Rev. D 93, 052014 (2016)

26. DarkSide Collaboration, P. Agnes, et al., Results from the first use of low radioactivity argon in a dark matter search. Phys. Rev. D 93, 081101 (2016) 